
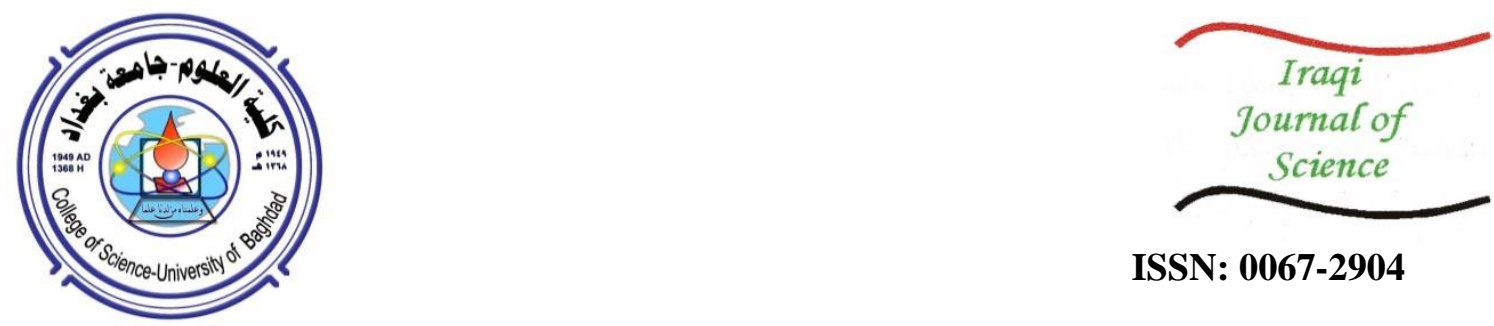

ISSN: 0067-2904

\title{
Differential Subordination and Superordination for Multivalent Functions Associated with Generalized Fox-Wright Functions
}

\author{
Zainab H.Mahmood ${ }^{1}$, Kassim A. Jassim² ${ }^{2}$, Buthyna N.Shihab ${ }^{3}$ \\ ${ }^{1}$ Department of Physical, College of Science, University of Baghdad, Baghdad, Iraq \\ ${ }^{2}$ Department of Mathematics, College of Science, University of Baghdad, Baghdad, Iraq \\ ${ }^{3}$ Department of Mathematics, College of Education for Pure Science Ibn Al Haitham, University of Baghdad, \\ Baghdad, Iraq
}

Received: 18/2/2021 Accepted: 22/4/2021

\begin{abstract}
In this paper, we derive some subordination and superordination results for certain subclasses of $\mathrm{p}^{-}$valent analytic functions that defined by generalized Fox-wright functions using the principle of differential subordination, ---------producing best dominant univalent solutions. We have also derived inclusion relations and solved majorization problem.
\end{abstract}

Keywords: analytic function, Univalent function, Multivalent function, Differential subordination, Fox-wright functions.

\section{التابعية التفاضلية والتفاضلية العليا لدوال متعددة التكافؤ مرتبطة مع دوال Fox-Wright المعمة}

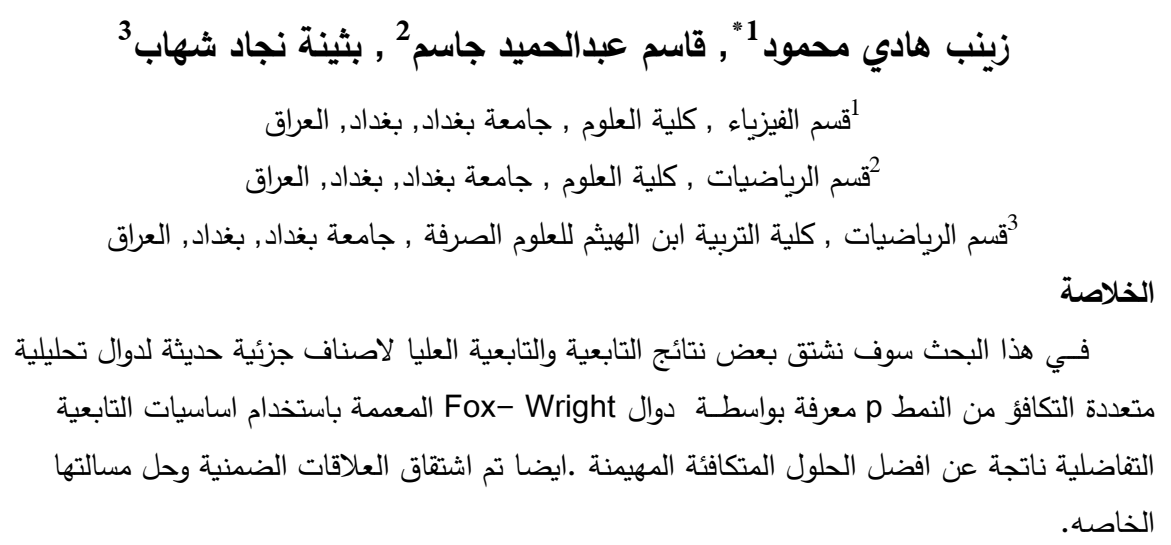

\section{Introduction}

Let $\triangle=\{\omega \in \mathbb{C}:|\omega|<1\}$ be the open unit disk in the complex plane $\mathbb{C}$, and $\mu=\mu(\triangle)$ is the class of analytic functions defined in $\triangle$. For a positive integer $n$ and a $\in \mathbb{C}$, let $\mu[a, n]=$ $\left\{f \in \mu: f(\omega)=\mathrm{a}+a_{n} \omega^{n}+a_{n+1} \omega^{n+1}+\cdots\right\}$, with $\mu_{0}=\mu[0,1], \mu=\mu[1,1]$.

Miller and Mocanu [1] assumed that $f$ and $g$ are functions of $\mu$. The function $f$ is said to be subordinate to $g$, written $f \prec \mathrm{g}$ or $f(\omega) \prec \mathrm{g}(\omega)$, if there exists a Schwarz function $\mathrm{w}(\omega)$ 
analytic in $\triangle$, with $w(0)=0$ and $|w(\omega)|<1$ such that $f(\omega)=g(w(\omega)),(\omega \in \triangle)$.In particular, if the function $g$ is univalent in $\triangle$, then $f<g$ if and only if $f(0)=g(0)$ and $f(\triangle) \subset g(\triangle)$.

Let $\psi: \mathbb{C}^{3} \times \triangle \rightarrow \mathbb{C}$, and $h$ is univalent in $\triangle$. If $f$ is analytic in $\triangle$ and satisfies the ( second -order) differential subordination

$$
\psi\left(f(\omega), \omega f^{\prime}(\omega), \omega^{2} f^{\prime \prime}(\omega) ; \omega\right) \prec h(\omega)
$$

then $f$ is called a solution of the differential subordination. The univalent function $q$ is called a dominant of the solutions of the differential subordination, or more simply dominant if $f<q$ for all $f$ satisfying (1.1) A dominant $\hat{q}$ that satisfies $\hat{q}<q$ for all dominants $q$ of (1.1) is said to be the best dominant of (1.1) .

Definition1.1: For the parameters $a_{i}, b_{j} \in \mathbb{C}$ and $A_{i}, B_{j} \in \mathcal{R}(i=1,2, \ldots, p ; j=1,2, \ldots, q)$, with $\quad \frac{a_{i}}{A_{i}} \neq 0,-1,-2, \ldots,(i=1,2, \ldots, p)$ and $\quad \frac{b_{i}}{B_{j}} \neq 0,-1,-2, \ldots,(j=1,2, \ldots, q)$, the generalized Fox-Wright function ${ }_{p} \Psi_{q}$ is defined by

$$
\begin{aligned}
& { }_{p} \Psi_{q}\left[\begin{array}{c}
\left(a_{i} ; A_{i}\right)_{1, p} \\
\left(b_{j}, B_{j}\right)_{1, q} ;
\end{array}\right]:={ }_{p} \Psi_{q}\left[\begin{array}{c}
\left(a_{1}, A_{1}\right), \ldots \ldots\left(a_{p}, A_{q}\right) \\
\left(B_{1}, b_{1}, \ldots \ldots,\left(b_{q}, B_{q}\right) ; \omega\right.
\end{array}\right]= \\
& \sum_{n=0}^{\infty} \frac{\prod_{i=1}^{p} \Gamma\left(a_{i}+n A_{i}\right)}{\prod_{j=1}^{q} \Gamma\left(b_{j}+n B_{j}\right)(1)_{n}} \omega^{n}, \quad(1.2)
\end{aligned}
$$

for suitable bounded values of $\omega \in \mathbb{C}$, where $\Gamma$ is the Gamma function. For more details see [2-5].

\section{Remark1.1:}

1. According to [6]. If $\sum_{j=1}^{q} B_{j}-\sum_{i=1}^{p} A_{i}>-1$, then the power series of (1.2) converges absolutely on $\mathrm{C}$ and the above defined function ${ }_{p} \Psi_{q}$ is an entire function. If $\sum_{j=1}^{q} B_{j}-$ $\sum_{i=1}^{p} A_{i}=-1$, then the power series of (1.2) converges absolutely on the disk $|\omega|<\frac{\prod_{j=1}^{q}\left|B_{j}\right|^{B_{j}}}{\prod_{i=1}^{p}\left|A_{i}\right|^{A_{i}}}$. 2.If $A_{i}=(i=1,2, \ldots \ldots, p)$ and $B_{j}=1(j=1,2, \ldots, q)$, one can find as the relationship [7]

$$
\Omega\left(\begin{array}{l}
a_{1}, \ldots, a_{p} \\
b_{1}, \ldots, b_{q}
\end{array}\right) \cdot \Psi_{q}\left[\begin{array}{cc}
\left(a_{i}, 1\right)_{1, p} & \\
\left(b_{j}, 1\right)_{1, q} ; & \omega
\end{array}\right]={ }_{p} \mathcal{F}_{q}\left(\begin{array}{cc}
a_{1}, \ldots, a_{p} \\
b_{1}, \ldots, b_{q} ; & \omega
\end{array}\right)
$$

where ${ }_{p} \mathcal{F}_{q}\left(\begin{array}{c}a_{1}, \ldots, a_{p} \\ b_{1}, \ldots, b_{q} ; \quad \omega\end{array}\right)$ is the generalized hypergeometric function [4], and

$$
\Omega=\Omega\left(\begin{array}{l}
a_{1}, \ldots, a_{p} \\
b_{1}, \ldots, b_{q}
\end{array}\right):=\frac{\prod_{j=1}^{q} \Gamma\left(b_{j}\right)}{\prod_{i=1}^{p} \Gamma\left(a_{i}\right)}
$$

3. For $p=1, q=1, A_{1}=1$ and $B_{1}=1$, the Fox-Wright defined function ${ }_{p} \Psi_{q}$ reduces to

$$
{ }_{1} \Psi_{1}\left[\begin{array}{cc}
(a, 1)_{1,1} & \\
(b, 1)_{1,1} ; & \omega
\end{array}\right]=\sum_{n=0}^{\infty} \frac{\Gamma(a+n)}{\Gamma(b+n)(1)_{n}} \omega^{n}, \omega \in \triangle .
$$

4. With the parameters values $a=1$ and $b=a>0(b \geq a)$, the function ${ }_{1} \Psi_{1}$ represents the classical Mittag-Leffler function for example see [8]. Moreover ${ }_{\mathrm{p}} \Psi_{\mathrm{q}}$ is a special case of the Fox's $\mathfrak{H}$-function $\mathcal{H}_{k, l}^{m, n}$ [3].

Use the generalized hypergeometric function, the authors [9] introduced a linear operator which was subsequently extended in [7] by using the Fox-Wright generalized hypergeometric 
function. Let

$\Theta\left[\begin{array}{l}\left(a_{i}, A_{i}\right)_{1, p} \\ \left(b_{j}, B_{j}\right)_{1, q}\end{array}\right]: D_{p} \rightarrow D_{p}$ be a linear operator which is defined by

$$
\Theta\left[\begin{array}{l}
\left(a_{i}, A_{i}\right)_{1, p} \\
\left(b_{j}, B_{j}\right)_{1, q}
\end{array}\right] f(\boldsymbol{\omega}):=\boldsymbol{\omega}_{\boldsymbol{p}} \boldsymbol{\phi}_{\boldsymbol{q}}\left[\begin{array}{cc}
\left(a_{i}, A_{i}\right)_{1, p} \\
\left(b_{j}, B_{j}\right)_{1, q} ;
\end{array}\right] * f(\boldsymbol{\omega}) .
$$

Note that for $f(\omega)$ of the form (1.1)and $\phi_{q}=\Omega_{p} \Psi_{p}$, t one can obtain that

$$
\Theta\left[\begin{array}{l}
\left(a_{i}, A_{i}\right)_{1, p} \\
\left(b_{j}, B_{j}\right)_{1, q}
\end{array}\right] f(\boldsymbol{\omega}):=\boldsymbol{\omega}+\sum_{n=2}^{\infty} \boldsymbol{\delta}_{n}\left(\boldsymbol{a}_{1}\right) \boldsymbol{a}_{n} \boldsymbol{\omega}^{n},
$$

where $\boldsymbol{\delta}_{\boldsymbol{n}}\left(\boldsymbol{a}_{\boldsymbol{i}}\right)$ is given by

and $\Omega$ is given by (1.3).

$$
\boldsymbol{\delta}_{\boldsymbol{n}}\left(\boldsymbol{a}_{\boldsymbol{i}}\right)=\frac{\Omega \Gamma\left(a_{1}+(n-1) A_{1}\right) \ldots . \Gamma\left(a_{p}+(n-1) A_{p}\right)}{\Gamma\left(b_{1}+(n-1) B_{1}\right) \ldots \Gamma\left(b_{q}+(n-1) B_{q}\right)}
$$

For convenience propose the contracted notation $\Theta\left(a_{i}\right) f(\boldsymbol{\omega})$ can be represented as follows:

$$
\Theta\left(a_{i}\right) f(\boldsymbol{\omega})=\boldsymbol{\Theta}\left[\begin{array}{c}
\left(a_{1}, A_{1}, \ldots \ldots .\left(a_{p}, A_{q}\right)\right. \\
\left(B_{1}, b_{1}\right) \ldots \ldots .\left(b_{q}, B_{q}\right)
\end{array}\right] f(\boldsymbol{\omega})
$$

From the equation (1.4) one can get the recursive relation that involves the operator $\Theta\left(a_{i}\right) f(\boldsymbol{\omega})$

$$
A_{i} \omega\left(\Theta\left(a_{i}\right) f(\boldsymbol{\omega})\right)^{\prime}=a_{i} \Theta\left(a_{i}+1\right) f(\boldsymbol{\omega})-\left(a_{i}-A_{i}\right) \Theta\left(a_{i}\right) f(\boldsymbol{\omega})
$$

Note that there are several interesting operators that are special cases of the linear operator (1.4). They have been extensively studied by researchers in [10-15], Hohlov operator and others. For more details see[16-18].

In order to prove the main results, we need the following definitions and theorem.

Definition 1.7.[19] The set of all function $q$ hat, which is denoted by $\mathrm{Q}$, are analytic and injective on $\bar{\triangle} \backslash E(q)$, where

$$
E(q)=\left\{\xi \in \partial \triangle: \lim _{\omega \rightarrow \xi} q(\omega)=\infty\right\}
$$

such that $q^{\prime}(\xi) \neq 0$ for $\xi \in \partial \Delta \backslash E(q)$, Furthermore let $(a), Q(0) \equiv Q_{0}$ and $Q(1)=Q_{1}$ such that $q(0)=a$.

Definition 1.8[19]. Let $\Omega$ be a subset of $\mathbb{C}, q \in Q$ and $n$ is a positive integer. The class of admissible functions $\psi[\Omega, q]$ consists function $\psi: \mathbb{C}^{3} \times \triangle \rightarrow \mathbb{C}$ that satisfies the admissibility condition:

whenever

$$
\psi(r, \mathcal{S}, v ; \omega, \xi) \notin \Omega \text {, }
$$

$$
\begin{aligned}
& r=q(\xi), \mathcal{S}=k \xi q^{\prime}(\xi), \text { and } \\
& \Re\left\{\frac{\mathrm{t}}{\mathcal{S}}+1\right\} \geq k \Re\left\{\frac{\xi \mathrm{q}^{\prime \prime}(\xi)}{\mathrm{q}^{\prime}(\xi)}+1\right\},
\end{aligned}
$$

where $\omega \in \triangle, \xi \in \partial \triangle \backslash E(q)$ and $k \geq n$. We write $\psi_{1}[\Omega, q]=\psi[\Omega, q]$. In addition if $\omega \in \triangle, \xi \in \partial \triangle$ and $b \geq n \geq 1$, then in particular we write $\psi_{1}^{\prime}[\Omega, q]=\psi^{\prime}[\Omega, q]$.

Theorem 1.1[19].Let $\Psi \in \psi_{n}[\Omega, q]$, with $\quad(0)=a$. If the analytic function $F \in$ $\mu[a, n]$ satisfies $\Psi\left(g(\omega), \omega g^{\prime}(\omega), \omega^{2} g^{\prime \prime}(\omega) ; \omega\right) \in \Omega$, then $F(\omega) \prec q(\omega)$.

\section{Main Results}

Definition 2.1: Let $\Omega$ be a subset of $\mathbb{C}$ with $q \in \mathrm{Q}_{0} \cap \mathcal{H}_{0}$. The class of admissible functions $\Phi_{k}[\Omega, q]$ consists of those functions $\emptyset: \mathbb{C}^{3} \times \triangle \times \bar{\triangle} \rightarrow \mathbb{C}$ which satisfies the admissibility condition: 
where

$$
\emptyset(u, v, w ; \omega, \xi) \notin \Omega
$$

$$
u=q(\xi), v=\frac{k A_{i} \xi q^{\prime}(\xi)-\left(A_{i}-a_{i}\right) q(\xi)}{a_{i}},\left(a_{i} \in \mathbb{C} \backslash \mathbb{Z}_{0}^{-}, a_{i} \neq 0\right),
$$

and

$$
\begin{gathered}
\Re\left\{\frac{\omega a_{i}\left(a_{i}+1\right)-a_{i}\left(2 a_{i}-2 A_{i}+1\right) v+\left(A_{i}-a_{i}\right)^{2} u}{A_{i}\left(a_{i} v+\left(A_{i}-a_{i}\right) u\right)}\right\} \geq k \mathfrak{R}\left\{\frac{\xi \mathrm{q}^{\prime \prime}(\xi)}{\mathrm{q}^{\prime}(\xi)}+1\right\}, \\
\omega \in \triangle, \zeta \in \partial \triangle \backslash E(q), \xi \in \bar{\triangle} \text { and } k \geq 1 .
\end{gathered}
$$

Theorem 2.1: Let $\emptyset \in \Phi_{k}[\Omega, q]$. If $f \in \not_{p}$ satisfies

$$
\begin{gathered}
\left\{\phi\left(\Theta\left(a_{i}\right) f(\omega)\right), \Theta\left(a_{i}+1\right) f(\omega), \Theta\left(a_{i}+2\right) f(\omega) ; \omega \in \triangle\right\} \subset \Omega \\
\Theta\left(a_{i}\right) f(\omega) \prec q(\omega) .
\end{gathered}
$$

Proof: From equation (1.6) we have

Which is equivalent to

$$
A_{i} \omega\left(\Theta\left(a_{i}\right) f(\boldsymbol{\omega})\right)^{\prime}=a_{i} \Theta\left(a_{i}+1\right) f(\boldsymbol{\omega})-\left(a_{i}-A_{i}\right) \Theta\left(a_{i}\right) f(\boldsymbol{\omega})
$$

$$
\Theta\left(a_{i}+1\right) f(\boldsymbol{\omega})=\frac{A_{i} \omega\left(\Theta\left(a_{i}\right) f(\boldsymbol{\omega})\right)^{\prime}+\left(a_{i}-A_{i}\right) \Theta\left(a_{i}\right) f(\boldsymbol{\omega})}{a_{i}} .
$$

Now assume that $F(\omega)=\Theta\left(a_{i}\right) f(\boldsymbol{\omega})$, then

Therefore,

$$
\Theta\left(a_{i}+1\right) f(\boldsymbol{\omega})=\frac{A_{i} \omega F^{\prime}(\omega)+\left(a_{i}-A_{i}\right) F(\omega)}{a_{i}} .
$$

and from equation (2.4), we have

$$
\Theta\left(a_{i}+2\right) f(\boldsymbol{\omega})=\frac{A_{i}^{2} \omega^{2} F^{\prime \prime}(\omega)+A_{i}\left(2 a_{i}-A_{i}+1\right) \omega F^{\prime}(\omega)+\left(a_{i}-A_{i}\right)\left(a_{i}-A_{i}-1\right) \boldsymbol{F}(\boldsymbol{\omega})}{a_{i}\left(a_{i}+1\right)}
$$

So that

$$
\left(\Theta\left(a_{i}+1\right) f(\boldsymbol{\omega})\right)^{\prime}=\frac{A_{i} \omega F^{\prime \prime}(\omega)+a_{i} F^{\prime}(\omega)}{a_{i}}
$$

$$
\begin{aligned}
\Theta\left(a_{i}+2\right) f(\boldsymbol{\omega}) & \\
= & \frac{1}{a_{i}}\left[\frac{A_{i} \omega\left(A_{i} \omega F^{\prime \prime}(\omega)+a_{i} F^{\prime}(\omega)\right)}{\left(a_{i}+1\right)}\right. \\
& \left.+\left(a_{i}+1-A_{i}\right)\left(\frac{A_{i} \omega F^{\prime}(z)+\left(a_{i}-A_{i}\right) F(\omega)}{\left(a_{i}+1\right)}\right)\right] \\
= & \frac{A_{i}{ }^{2} \omega^{2} F^{\prime \prime}(\omega)+A_{i}\left(2 a_{i}+1-A_{i}\right) \omega F^{\prime}(z)+\left(A_{i}-a_{i}\right)\left(A_{i}-a_{i}-1\right) F(\omega)}{a_{i}\left(a_{i}+1\right)}
\end{aligned}
$$

Let $u=r \quad, v=\frac{A_{i} \mathcal{S}-\left(A_{i}-a_{i}\right) r}{a_{i}}, \quad w=\frac{A_{i}{ }^{2} \mathrm{t}+A_{i}\left(2 a_{i}+1-A_{i}\right) \mathcal{S}+\left(A_{i}-a_{i}\right)\left(A_{i}-a_{i}-1\right) r}{a_{i}\left(a_{i}+1\right)}$

and

let $\psi(r, \mathcal{S}, \mathrm{t} ; \omega, \xi)=\emptyset(u, v, w ; \omega, \xi)=$

$\varnothing\left(r, \frac{A_{i} \mathcal{\delta}-\left(A_{i}-a_{i}\right) r}{a_{i}}, \frac{A_{i}{ }^{2} \mathrm{t}+A_{i}\left(2 a_{i}+1-A_{i}\right) \mathcal{S}+\left(A_{i}-a_{i}\right)\left(A_{i}-a_{i}-1\right) r}{a_{i}\left(a_{i}+1\right)} ; \omega\right)$.

So that by equation (2.4) and (2.5), we obtain

$$
\psi\left(F(\omega), \omega F^{\prime}(\omega), \omega^{2} F^{\prime \prime}(\omega) ; \omega, \xi\right)=\varnothing\left(\left(\Theta\left(a_{i}\right) f(\omega)\right), \Theta\left(a_{i}+1\right) f(\omega), \Theta\left(a_{i}+\right.\right.
$$

2) $f(\omega) ; \omega)$. 
by using equation (2.3), we get

We also use the following equation

$$
\psi\left(F(\omega), \omega F^{\prime}(\omega), \omega^{2} F^{\prime \prime}(\omega) ; \omega, \xi\right) \in \Omega
$$

$$
w=\frac{A_{i}{ }^{2} \omega^{2} F^{\prime \prime}(\omega)+A_{i}\left(2 a_{i}+1-A_{i}\right) \omega F^{\prime}(z)+\left(A_{i}-a_{i}\right)\left(A_{i}-a_{i}-1\right) F(\omega)}{a_{i}\left(a_{i}+1\right)},
$$

and by simple calculations we get

$$
\frac{\omega a_{i}\left(a_{i}+1\right)-a_{i}\left(2 a_{i}-2 A_{i}+1\right) v+\left(A_{i}-a_{i}\right)^{2} u}{A_{i}\left(a_{i} v+\left(A_{i}-a_{i}\right) u\right)}=\frac{\mathrm{t}}{\mathcal{S}}+1 .
$$

We note that the admissibility condition for $\emptyset \in \Phi_{k}[\Omega, q]$ is equivalent to the admissibility condition for $\psi$, then $F(\omega) \prec q(\omega)$.

Example 2.1. Let the class of $\Phi_{k v}[\Omega, q]$ consists of those functions $\emptyset: \mathbb{C}^{3} \times \triangle \rightarrow \mathbb{C}$ that satisfies the admissibility condition:

$$
v=\frac{A_{i} k \xi q^{\prime}(\xi)+\left(A_{i}-a_{i}\right) q(\xi)}{a_{i}} \notin \Omega
$$

$\boldsymbol{\omega} \in \triangle, \zeta \in \partial \triangle \mid E q$, andm $\geq p . I \in D_{\rho}$ satisfies $\mathcal{S}_{0, \omega}^{\lambda, \mu, \eta} f(\omega) \subset \mathbf{\Omega}$, then

$$
\left(\Theta\left(a_{i}\right) f(\omega)\right) \prec q(\omega)
$$

We consider the special situation when $\Omega \neq \mathbb{C}$ is a simply connected domain. Now if we assume that $\Omega \neq \mathbb{C}$ be a simply connected, so that $\Omega=h(\triangle)$ for some conformal mapping $h$ of $\triangle$ onto $\Omega$. The next result is an abrupt outcome of Theorem 2.1.

Theorem2.2 :Let $\emptyset \in \Phi_{k}[h, q]$.If $f \in \not_{p}$ satisfies

$$
\varnothing\left(\left(\Theta\left(a_{i}\right) f(\omega)\right), \Theta\left(a_{i}+1\right) f(\omega), \Theta\left(a_{i}+2\right) ; \omega \in \triangle\right) \prec \mathrm{h}(\omega),
$$

Then

$$
\Theta\left(a_{i}\right) f(\omega) \prec q(\omega) .
$$

The next result is an extension of Theorem (2.1) to the case where the behavior of $q$ on $\triangle$ is not known.

Corollary 2.1.Let $\Omega \subset \mathbb{C}, q$ be univalent in $\triangle$ and $q(0)=0$.Let $\emptyset \in \Phi_{k}\left[\Omega, q_{\rho}\right]$ for some $\rho \in$ $(0,1)$, where $q_{\rho}(\omega)=q(\rho \omega)$. If $f \in \not_{\rho}$ satisfies

$$
\varnothing\left(\left(\Theta\left(a_{i}\right) f(\omega)\right), \Theta\left(a_{i}+1\right) f(\omega), \Theta\left(a_{i}+2\right) ; \omega \in \triangle\right) \in \Omega,
$$

Then

$$
\left(\Theta\left(a_{i}\right) f(\omega)\right) \prec q(\omega)
$$

Proof. From Theorem 2.1,we have $\left(\Theta\left(a_{i}\right) f(\omega)\right), \prec q_{p}(\omega)$.

Theorem 2.3. Let $h$ and $q$ are univalent .Also, $q(0)=0, q_{\rho}(\omega)=q(\rho \omega)$ and $h_{\rho}(\omega)=h(\rho \omega)$.

Let $\emptyset: \mathbb{C}^{3} \times \triangle \times \bar{\triangle} \rightarrow \mathbb{C}$ satisfies one of the following conditions:

(1) $\varnothing \in \Phi_{k}\left[\Omega, q_{\rho}\right]$ for some $\rho \in(0,1)$ or

(2) There exists $\rho_{0} \in(0,1)$ such that $\emptyset \in \Phi_{\mathrm{k}}\left[h_{\rho}, q_{\rho}\right]$ for all $\rho \in\left(\rho_{0}, 1\right)$.

If $f \in \mathcal{D} \rho$ satisfies (2.12), then

$$
\left(\Theta\left(a_{i}\right) f(\omega)\right) \prec q(\omega) .
$$

Proof:Case (1): By using Theorem (2.1), we get $\left(\Theta\left(\mathrm{a}_{i}\right) f(\omega)\right), \prec q_{\rho}(\omega)$. Since $q_{\rho}(\omega) \prec$ $q(\omega)$, we deduce

$$
\left(\Theta\left(\mathrm{a}_{i}\right) f(\omega)\right) \prec q(\omega) .
$$

Case (2): Assume that $F(\omega)=\left(\Theta\left(\mathrm{a}_{i}\right) f(\omega)\right)$, and $F_{\rho}(\omega)=F(\rho \omega)$. So that $\varnothing\left(F_{\rho}(\omega), \omega F_{\rho}^{\prime}(\omega), \omega^{2} F_{\rho}^{\prime \prime}(\omega) ; \rho \omega\right)=\varnothing\left(F(\rho \omega), \rho \omega F^{\prime}(\rho \omega), \rho^{2} \omega^{2} F^{\prime \prime}(\rho \omega) ; \rho \omega\right) \in h_{\rho}(\triangle)$.

By using Theorem 2.1 with associated $\varnothing\left(F(\omega), \omega F^{\prime}(\omega), \omega^{2} F^{\prime \prime}(\omega) ; w(\omega)\right) \in \Omega$, where $w$ is any function mapping from 
$\triangle$ onto $\triangle$, with $w(\omega)=\rho \omega$, we obtain $F_{\rho}(\omega) \prec q_{\rho}(\omega)$ for $\rho \in\left(\rho_{0}, 1\right)$. By letting $\rho \rightarrow 1^{-}$,we $\operatorname{get} F(\omega) \prec q(\omega)$.

Therefore

$$
\left(\Theta\left(\mathrm{a}_{i}\right) f(\omega)\right) \prec q(\omega)
$$

The next theorem gives the best dominant of the differential subordination (2.12).

Theorem2.4. Let $h$ be univalent in $\triangle$ and let $\emptyset: \mathbb{C}^{3} \times \Delta \times \bar{\triangle} \rightarrow \mathbb{C}$. Suppose that the differential equation

$$
\begin{aligned}
& \varnothing\left(q(\omega), \frac{\mathrm{A}_{i} \omega q^{\prime}(\omega)+\left(\mathrm{A}_{i}-\mathrm{a}_{i}\right) q(\omega)}{\mathrm{a}_{i}}, \frac{\mathrm{A}_{i}^{2} \omega^{2} q^{\prime \prime}(\omega)+\mathrm{A}_{i}\left(2 \mathrm{a}_{i}+1-\mathrm{A}_{i}\right) \omega q^{\prime}(\omega)+\left(\mathrm{A}_{i}-\mathrm{a}_{i}\right)\left(\mathrm{A}_{i}-\mathrm{a}_{i}-1\right) q(\omega)}{\mathrm{a}_{i}\left(\mathrm{a}_{i}+1\right)} ; \omega\right) \\
& =h(\omega),
\end{aligned}
$$

has a solution $q$ with $q(0)=0$ and satisfies one of the following conditions:

(1) $q \in Q_{0}$ and $\emptyset \in \Phi_{\mathrm{k}}[h, q]$.

(2) $q$ is univalent in $\triangle$ and $\varnothing \in \Phi_{\mathrm{k}}\left[h, q_{\rho}\right]$ for some $\rho \in(0,1)$, or

(3) $q$ is univalent in $\triangle$ and there exists $\rho_{0} \in(0,1)$ such that $\varnothing \in \Phi_{\mathrm{k}}\left[h_{\rho}, q_{\rho}\right]$, for all $\rho_{0} \in$ $(0,1)$.If $f \in \mathcal{D} \rho$ satisfies (2.12), then $\left(\Theta\left(\mathrm{a}_{i}\right) f(\omega)\right),<q(\omega)$ and $q$ is the bestdominant.

Proof. By using Theorem 2.2 and Theorem 2.3, we get that $q$ is a dominant of (2.12). Since $q$ satisfies (2.14), it is also a solution of (2.12) and therefore $q$ will be dominant by all dominants of (2.12). Hence $q$ is the best dominant of (2.12).

Definition 2.2.Let $\Omega$ be a set in $\mathbb{C}$ and $M>0$. The class of admissible functions $\Phi_{k}[\Omega, q]$ consists of functions $\emptyset: \mathbb{C}^{3} \times \triangle \times \bar{\triangle} \rightarrow \mathbb{C}$ such that

$$
\begin{aligned}
& \emptyset\left(M e^{i \theta}, \frac{\left(\mathrm{A}_{i} k-\left(\mathrm{A}_{i}-\mathrm{a}_{i}\right)\right) M e^{i \theta}}{\mathrm{a}_{i}}, \frac{\mathrm{A}_{i}{ }^{2} L+\left[\mathrm{A}_{i} k\left(2 \mathrm{a}_{i}-\mathrm{A}_{i}+1\right)+\left(\mathrm{A}_{i}-\mathrm{a}_{i}\right)\left(\mathrm{A}_{i}-\mathrm{a}_{i}-1\right)\right] M e^{i \theta}}{\mathrm{a}_{i}\left(\mathrm{a}_{i}+1\right)} ; \omega\right) \\
& \notin \Omega,
\end{aligned}
$$

Where $\lambda>0, \theta \in \mathcal{R}, \mathcal{R}\left(L e^{i \theta}\right) \geq k(k-1)$ Mfor all real $\theta, k \geq 1, \omega \in \triangle$.

Corollary 2.2: Let $\emptyset \in \Phi_{k}[\Omega, M]$. If $f \in \mathcal{D} \rho$ satisfies that

$$
\varnothing\left(\left(\Theta\left(a_{i}\right) f(\omega)\right), \Theta\left(a_{i}+1\right) f(\omega), \Theta\left(a_{i}+2\right) ; \omega\right) \in \Omega \text {, then } \Theta\left(a_{i}\right) f(\omega) \prec M \omega \text {. }
$$

Corollary 2.3: Let $\emptyset \in \Phi_{k}[\Omega, M]$. If $f \in \mathcal{D} \rho$ satisfies that

$$
\left|\left(\Theta\left(\mathrm{a}_{i}\right) f(\omega)\right), \Theta\left(\mathrm{a}_{i}+1\right) f(\omega), \Theta\left(\mathrm{a}_{i}+2\right) ; \omega\right|<M \text {, then }\left|\Theta\left(\mathrm{a}_{i}\right) f(\omega)\right|<M
$$

Corollary 2.4:LetM $>0, \mathrm{a}_{i} \in \mathbb{C} \backslash \mathbb{Z}_{0}^{-}, \mathcal{R}\left(\mathrm{a}_{i}\right) \geq 0$ and $f \in \mathcal{D} \rho$ satisfies the following inequality

$$
\left|\Theta\left(\mathrm{a}_{i}+1\right) f(\omega)\right|<M \text {, then }\left|\Theta\left(\mathrm{a}_{i}+1\right) f(\omega)\right|<M, \omega \in \triangle \text {. }
$$

Proof. From Corollary (2.2) one can take $\emptyset(u, v, w, \omega)=v=,\frac{\left(A_{i} k-\left(A_{i}-a_{i}\right)\right) M e^{i \theta}}{a_{i}}$

Corollary 2.5.If $\mathrm{M}>0, \mathrm{a}_{i} \in \mathbb{C} \backslash \mathbb{Z}_{0}^{-}$, If $f \in \mathcal{D} \rho$ satisfies the following inequality

$$
\left|\Theta\left(\mathrm{a}_{i}+1\right) f(\omega)-\left(\frac{\mathrm{A}_{i}}{\mathrm{a}_{i}}-1\right) \Theta\left(\mathrm{a}_{i}\right) f(\omega)\right|<\frac{M \mathrm{~A}_{i}}{\mathrm{a}_{i}}
$$

then $\left|\Theta\left(\mathrm{a}_{i}\right) f(\omega)\right|<M,, \boldsymbol{\omega} \in \triangle$.

Proof. Let $\varnothing(u, v, w, \omega)=,v+\left(\frac{A_{i}}{a_{i}}-1\right) u$ and $\Omega=h(\triangle)$ where $h(\boldsymbol{\omega})=\frac{M A_{i}}{a_{i}} \boldsymbol{\omega}, M>0$.

From the corollary (1.3), it is enough to show that $\phi \in \Phi_{k}[\Omega, q]$, that means the admissibility condition (1.6) is satisfied. Hence,

$$
\begin{aligned}
& \left|\phi\left(M e^{i \theta}, \frac{\left(\mathrm{A}_{i} k-\left(\mathrm{A}_{i}-\mathrm{a}_{i}\right)\right) M e^{i \theta}}{\mathrm{a}_{i}}, \frac{\mathrm{A}_{i}^{2} L+\left[\mathrm{A}_{i} k\left(2 \mathrm{a}_{i}-\mathrm{A}_{i}+1\right)+\left(\mathrm{A}_{i}-\mathrm{a}_{i}\right)\left(\mathrm{A}_{i}-\mathrm{a}_{i}-1\right)\right] M e^{i \theta}}{\mathrm{a}_{i}\left(\mathrm{a}_{i}+1\right)} ; \omega\right)\right| \\
& \geq\left|\frac{\mathrm{A}_{i} M e^{i \theta}}{\mathrm{a}_{i}}\right| \geq \frac{M \mathrm{~A}_{i}}{\left|\mathrm{a}_{i}\right|}
\end{aligned}
$$

Whenever $\boldsymbol{\omega} \in \triangle$. $\theta \in \mathcal{R}, \mathrm{a}_{i} \in \mathbb{C} \backslash \mathbb{Z}_{0}^{-}, \mathrm{a}_{i} \neq-1$ and $k \geq 1$.

Definition 2.3.Let $\Omega$ be a set in $\mathbb{C}$ and $q \in Q_{0} \cap \mathcal{H}_{0}$. The class of admissible functions $\Phi_{k, 1}[\Omega, q]$ consists of $\emptyset: \mathbb{C}^{3} \times \triangle \rightarrow \mathbb{C}$ that satisfies 
whenever

$$
\emptyset(u, v, w, \omega, \xi) \notin \Omega,
$$

$$
u=q(\xi), v=\frac{1}{\mathrm{a}_{i}+1}\left(\frac{k \mathrm{~A} \xi q^{\prime}(\xi)}{q(\xi)}+\mathrm{a}_{i} q(\xi)+1\right), \mathrm{a}_{i} \in \mathbb{C} \backslash \mathbb{Z}_{0}^{-}
$$

and

$$
\begin{aligned}
& \Re\left\{\frac{v\left(\mathrm{a}_{i}+1\right)\left(\left(\mathrm{a}_{i}+1\right)(w-v)+w-1\right)}{\mathrm{A}_{i}\left(v\left(\mathrm{a}_{i}+1\right)-\mathrm{a}_{i} u-1\right)}+\frac{\left(v\left(\mathrm{a}_{i}+1\right)-\mathrm{a}_{i} u\left(\mathrm{~A}_{i}+1\right)-1\right)}{\mathrm{A}_{i}}\right\} \\
& \geq k \Re\left\{\frac{\xi q^{\prime \prime}(\xi)}{q^{\prime}(\xi)}+1\right\},
\end{aligned}
$$

$\omega \in \triangle, \zeta \in \partial \triangle \backslash E(q), k \geq 1$ and $\mathrm{a}_{i} \neq-1$.

Theorem 2.5. Let $\emptyset \in \Phi_{k}^{\prime}[h, q]$. If $f \in \mathcal{D} \rho, \Theta\left(\mathrm{a}_{i}\right) f(\omega) \in Q_{0}$ and

is univalent in $\triangle$,then

$$
\varnothing\left(\left(\Theta\left(a_{i}\right) f(\omega)\right), \Theta\left(a_{i}+1\right) f(\omega), \Theta\left(a_{i}+2\right) ; \omega\right)
$$

$\Omega \subset\left\{\varnothing\left(\left(\Theta\left(a_{i}\right) f(\omega)\right), \Theta\left(a_{i}+1\right) f(\omega), \Theta\left(a_{i}+2\right) ; \omega \in \triangle, \xi \in \bar{\triangle}\right)\right\}$ which implies that

$$
q(z) \prec \Theta\left(a_{i}\right) f(\omega) .
$$

Proof: By (11) and $\Omega \subset\left\{\varnothing\left(\left(\Theta\left(\mathrm{a}_{i}\right) f(\omega)\right), \Theta\left(\mathrm{a}_{i}+1\right) f(\omega), \Theta\left(\mathrm{a}_{i}+2\right) ; \omega \in \triangle, \xi \in \bar{\triangle}\right)\right.$, we have $\left.\Omega \subset\left\{\psi\left(F(\omega), \omega F^{\prime}(\omega), \omega^{2} F^{\prime \prime}(\omega) ; \omega, \xi\right) ; \omega \in \triangle, \xi \in \bar{\triangle}\right)\right\}$.

from

$$
\begin{aligned}
u=r, v= & \frac{A_{i} \mathcal{S}-\left(\mathrm{A}_{i}-\mathrm{a}_{i}\right) r}{\mathrm{a}_{i}}, w \\
& =\frac{\mathrm{A}_{i}{ }^{2} \mathrm{t}+\mathrm{A}_{i}\left(2 \mathrm{a}_{i}+1-\mathrm{A}_{i}\right) \mathcal{S}+\left(\mathrm{A}_{i}-\mathrm{a}_{i}\right)\left(\mathrm{A}_{i}-\mathrm{a}_{i}-1\right) r}{\mathrm{a}_{i}\left(\mathrm{a}_{i}+1\right)}
\end{aligned}
$$

we see that the admissibility for $\emptyset \in \Phi^{\prime}{ }_{k}[\Omega, q]$ is equivalent to admissibility condition for $\psi$. Hence, $\psi \in \Psi^{\prime}[\Omega, q]$ and so we have $q(z) \prec \Theta\left(a_{i}\right) f(\omega)$.

The following Theorem is immediately consequence of Theorem(2.5).

Theorem2.6. Let $q \in \mu[0, p], h$ be analytic in $\triangle$ and $\emptyset \in \Phi_{k}^{\prime}[h, q]$. If $f(\omega) \in \mathcal{D} \rho$,

and

$$
\Theta\left(a_{i}\right) f(\omega) \in Q_{0}
$$

It is univalent in $\triangle$, then

$$
\left\{\varnothing\left(\left(\Theta\left(a_{i}\right) f(\omega)\right), \Theta\left(a_{i}+1\right) f(\omega), \Theta\left(a_{i}+2\right) ; \omega\right\}\right.
$$

$$
h(\omega) \prec\left\{\varnothing\left(\left(\Theta\left(a_{i}\right) f(\omega)\right), \Theta\left(a_{i}+1\right) f(\omega), \Theta\left(a_{i}+2\right) ; \omega\right\},\right.
$$

which implies that $q(\omega) \prec \Theta\left(\mathrm{a}_{i}\right) f(\omega)$.

Theorem 2.7. Let $h$ be analytic in $\triangle$ and $\emptyset: \mathbb{C}^{3} \times \triangle \times \bar{\triangle} \rightarrow \mathbb{C}$.Suppose that

$$
\varnothing\left(\begin{array}{c}
\left.q(\omega), \frac{\mathrm{A}_{i} \omega q^{\prime}(\omega)+\left(\mathrm{A}_{i}-\mathrm{a}_{i}\right) q(\omega)}{\mathrm{a}_{i}}, \frac{\begin{array}{c}
\mathrm{A}_{i}{ }^{2} \omega^{2} q^{\prime \prime}(\omega)+\mathrm{A}_{i}\left(2 \mathrm{a}_{i}+1-\mathrm{A}_{i}\right) \omega q^{\prime}(\omega) \\
+\left(\mathrm{A}_{i}-\mathrm{a}_{i}\right)\left(\mathrm{A}_{i}-\mathrm{a}_{i}-1\right) q(\omega)
\end{array}}{\mathrm{a}_{i}\left(\mathrm{a}_{i}+1\right)} ; \omega\right) \\
=h(\omega),
\end{array}\right)
$$

has a solution $q \in Q_{0}$. If $\varnothing \in \Phi_{k}^{\prime}[h, q], f \in \mathcal{D} \rho, \Theta\left(\mathrm{a}_{i}\right) f(\omega) \in Q_{0}$ and $\emptyset\left\{\left(\Theta\left(a_{i}\right) f(\omega)\right), \Theta\left(a_{i}+1\right) f(\omega), \Theta\left(a_{i}+2\right) ; \omega\right\}$ is univalent in $\triangle$, then

$$
h(z) \prec \varnothing\left(\left(\Theta\left(a_{i}\right) f(\omega)\right), \Theta\left(a_{i}+1\right) f(\omega), \Theta\left(a_{i}+2\right) ; \omega\right\},
$$

implies that $q(z) \prec \Theta\left(\mathrm{a}_{i}\right) f(\omega)$, and $q$ is the best dominant.

Proof:The proof of this Theorem is the same as the proof Theorem (2.4). 
Theorem (2.2) and Theorem (2.6), we obtained the following Theorem.

Theorem 2.8 Suppose that $h_{1}$ and $q_{1}$ are analytic functions in $\triangle$, and $h_{2}$ is a univalent functions in $\triangle, q_{2} \in Q_{0}$ with $q_{1}(0)=q_{2}(0)=0$ with $\emptyset \in \Phi_{k}\left[h_{2}, q_{2}\right] \cap \Phi_{k}^{\prime}\left[h_{1}, q_{1}\right]$.

If $f \in \mathcal{D} \rho, \Theta\left(\mathrm{a}_{i}\right) f(\omega) \in \mu[0, p] \cap Q_{0}$ and

$\left\{\varnothing\left(\left(\Theta\left(\mathrm{a}_{i}\right) f(\omega)\right), \Theta\left(\mathrm{a}_{i}+1\right) f(\omega), \Theta\left(\mathrm{a}_{i}+2\right) ; \omega\right\}\right.$ is univalent in $\triangle$, then

$$
h_{1}(\omega) \prec \varnothing\left(\left(\Theta\left(a_{i}\right) f(\omega)\right), \Theta\left(a_{i}+1\right) f(\omega), \Theta\left(a_{i}+2\right) ; \omega\right\} \prec h_{2},
$$

And this implies that $q_{1}(\omega) \prec \Theta\left(\mathrm{a}_{i}\right) f(\omega) \prec q_{2}(\omega)$.

\section{Reference}

[1] Miller S.S., and Mocanu P.T. "Differential subordinations: Theory and Applications", Marcel Dekker Inc., New York, Basel, 2000.

[2] Srivastava, H., and M. Owa, S. "Some characterization and distortion theorems involving fractional calculus, generalized hypergeometric functions, Hadamard products, linear operators and certain subclasses of analytic functions", Nagoya Math. J., vol. 106, pp. 1-28, 1987.

[3] Srivastava H. M., and Monacha, H. L. "A treatise on generating functions", Ellis Horwood Series: Mathematics and its Applications, Horwood, Chichester, 1984.

[4] Srivastava H M., and Karlsson P. W. "Multiple Gaussian hypergeometric series", Ellis Horwood Series: Mathematics and its Applications, Horwood, Chichester, 1985.

[5] Srivastava. H. M., and Attiya A. "An integral operator associated with the Hurwitz-Lerch zeta function and differential subordination", Integral Transforms Spec. Funct, vol. 18, no. 3, pp. 207216, 2007.

[6] Kilbas A. A., Saigo M ., and Trujillo J. J. "On the generalized Wright function", Fract. Calc. Appl. Anal., vol. 5, no. 4, pp. 437-460, 2002.

[7] Dziok J., and Raina. R. K. "Families of analytic functions associated with the Wright generalized hypergeometric function", Demonstratio Math., vol. 37, no. 3, pp. 533-542, 2004.

[8] Erdelyi A et al. "Higher transcendental functions", McGraw-Hill, New York, vol. 3, 1956.

[9] Dziok J., and Srivastava H. M. "Classes of analytic functions associated with the generalized hypergeometric function", Appl. Math. Comput., vol. 103, 1-13, 1999.

[10] Dziok J., and Raina R. K. "Certain subclasses of analytic functions associated with the generalized hypergeometric function”, Integral Transforms Spec. Funct., vol. 14, no. 1, pp. 7-18, 2003.

[11] Carlson B. C., and Shaffer B. C. "Starlike and prestarlike hypergeometric functions", SIAM J. Math. Anal., vol. 15, no. 4, pp. 737-745, 1984.

[12] Ruscheweyh St. "New criteria for univalent functions", Proc. Amer. Math. Soc., vol. 49, pp. 109$115,1975$.

[13] Cho N. E., and Kim J. A. "Multiplier transformations and strongly close-to-convex functions", Bull. Korean Math. Soc., vol. 40, no. 3, pp. 399-410, 2003.

[14] Cho N. E., and Srivastava H. M. "Argument estimates of certain analytic functions defined by a class of multiplier transformations", Math. Comput. Modelling, vol. 37, no. 1-2, pp. 39-49, 2003.

[15] Choi J. H., Saigo M., and Srivastava H. M. "Some inclusion properties of a certain family of integral operators", J. Math. Anal. Appl., vol. 276, no. 1, pp. 432-445, 2003.

[16] Dziok J., Raina R. K., and Srivastava H. M. "Some classes of analytic functions associated with operators on Hilbert space involving Wright's generalized hypergeometric function", Proc. Jangjeon Math. Soc., vol. 7, no. 1, pp. 43-55, 2004.

[17] Waggas Galib Atshan A. H. Majeed and Kassim A. Jassim. "On a new class of univalent functions with application of fractional calculus operators defined by hohlov operator", Journal of Asian Scientific Research, vol. 4,no.2, 99-111, 2014.

[18] Rafid Habib Buti ., and Kassim A. Jassim. "On A Class of W-Valent Functions With Two Fixed Points Involving Hypergeomatric Function with Generalization Integral Operator", Iraqi Journal of Science, Vol. 60, no.8, pp.1753-1759, 2019.

[19] S. S. Miller and P. T. Mocanu, "Differential subordinations and univalent functions", Michigan Math. J. vol. 28, 157-171, 1981. 